\title{
Misdiagnosis related to premature diagnostic closure during the COVID-19 pandemic
}

\author{
Brandon Budhram MD, Alexandra O. Kobza MD, Naufal Mohammed MD
}

Cite as: CMAJ 2020 September 28;192:E1129-31. doi: 10.1503/cmaj.201426

CMAJ Podcasts: author interview at www.cmaj.ca/lookup/doi/10.1503/cmaj.201426/tab-related-content

A 40-year-old woman was admitted to the internal medicine service in April 2020 after a 10-day history of productive cough, exertional dyspnea, fatigue, chills and documented fevers. Her medical history was noncontributory. She was employed as a community personal support worker and had worked in long-term care facilities, none of which had reported an outbreak of coronavirus disease 2019 (COVID-19). She was unaware of any sick contacts or known exposure to someone infected with severe acute respiratory syndrome coronavirus 2 (SARS-CoV-2).

At presentation to the emergency department, the patient was febrile at $38.1^{\circ} \mathrm{C}$ and dyspneic with a respiratory rate of 28 breaths/min and oxygen saturation of $89 \%$ on room air. She had bilateral inspiratory crackles and her chest radiograph showed bilateral interstitial markings (Figure 1). Laboratory investigations were significant for a lymphopenia of 0.6 (normal range $1.5-4.0$ ) $\times 10^{9} / \mathrm{L}$ with a normal leukocyte count of 4.5 (normal range 4.0-11.0) $\times 10^{9} / \mathrm{L}$, hypoalbuminemia of 22 (normal range 35-50) g/L, elevated C-reactive protein (CRP) of 23.1 (nor$\mathrm{mal}<5.0) \mathrm{mg} / \mathrm{L}$, and an elevated lactate dehydrogenase (LDH) of 372 (normal range 100-220) units/L. A nasopharyngeal swab testing for multiple respiratory pathogens (including SARS-CoV-2) via multiplex polymerase chain reaction (PCR) was negative at presentation. Regardless, COVID-19 remained the leading diagnosis,

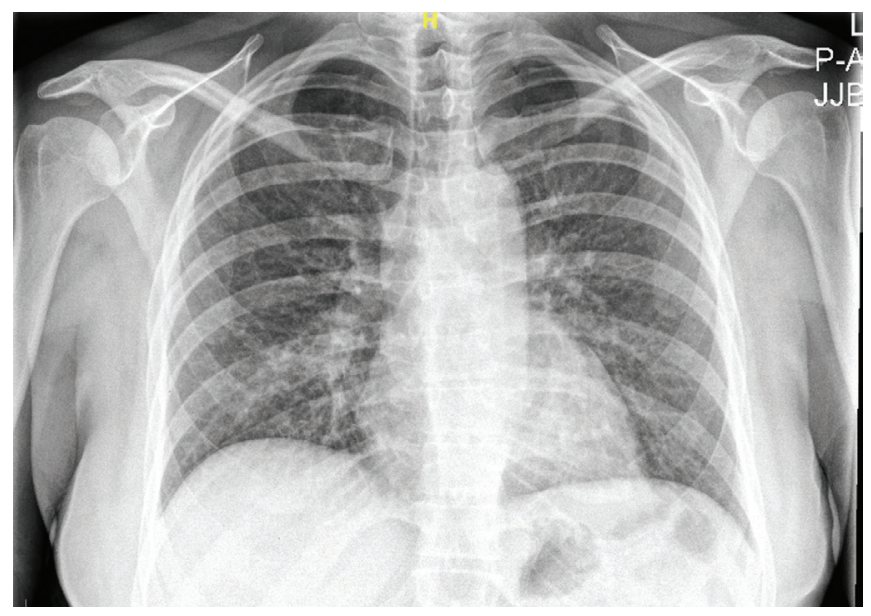

Figure 1: Chest radiograph of a 40-year-old woman, showing diffuse bilateral interstitial markings, slightly more prominent in the right perihilar distribution.

\section{KEY POINTS}

- There is a risk of premature diagnostic closure during the coronavirus disease 2019 (COVID-19) pandemic.

- In the absence of a confirmatory test, patients suspected of having COVID-19 require critical re-evaluation for other diagnoses at regular intervals.

- A thorough history and physical examination is still necessary in patients presenting with "classic" symptoms of COVID-19.

followed by other viral, bacterial and fungal pneumonias, with interstitial lung diseases being felt to be less likely.

We treated the patient empirically for community-acquired pneumonia with levofloxacin and her condition improved marginally over the next 72 hours with defervescence (on acetaminophen) and minor improvement in her oxygen saturation to $92 \%$. We sent a repeat nasopharyngeal swab and a sputum sample for PCR on day 3, specifically querying SARS-CoV-2, given the team's high suspicion for COVID-19; both were negative. We performed computed tomography (CT) of the chest on day 3 to assess for other infectious causes and interstitial lung diseases. It showed extensive bilateral ground-glass opacities (Figure 2).

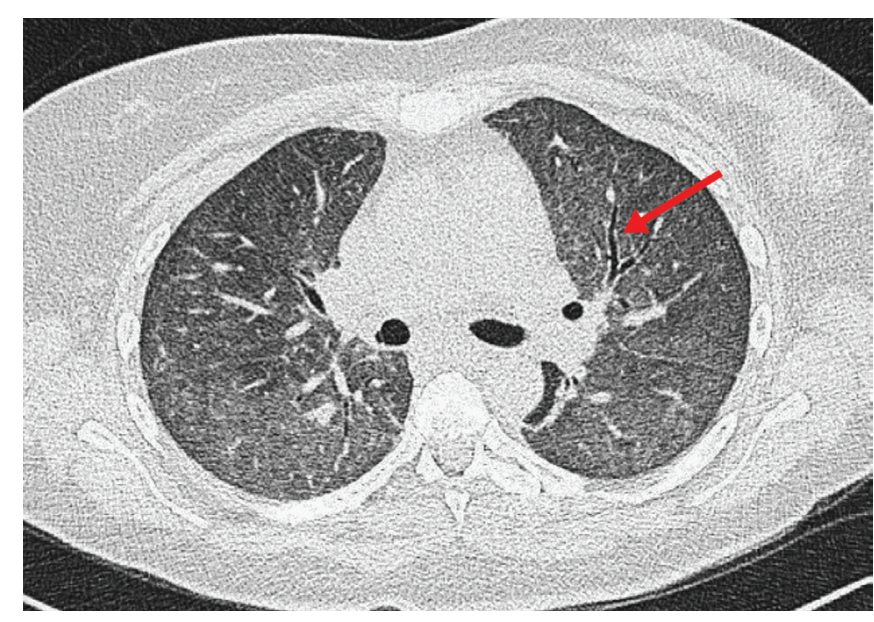

Figure 2: Computed tomography scan showing diffuse bilateral groundglass airspace opacities, further illustrated by the relatively darker, air-filled bronchus in the left upper lobe (arrow), known as the "dark bronchus" sign. 
Given the patient's CT findings, we consulted the respirology service and the patient underwent bronchoscopy on day 7 to rule out alternative diagnoses and establish a diagnosis of COVID-19. The procedure was significant for mild, diffuse bronchitis and oral candidiasis, for which we treated her with nystatin 500000 units, swish and swallow 4 times a day. The patient requested discharge from hospital after bronchoscopy and subsequently returned home with instructions to self-isolate while awaiting analysis of her bronchoscopy samples.

Two days later, the bronchoscopy samples tested negative on PCR for SARS-CoV-2, but positive for Pneumocystis jirovecii. The patient was readmitted to hospital for treatment for her $P$. jirovecii pneumonia, and to determine the cause of her underlying immunosuppression. A closer evaluation elicited multiple symptoms and signs not identified during her initial admission, including a 23-kg weight loss over 12 months; intermittent night sweats over 6 months; a violaceous, nonpruritic, painless plaque on her chest (which on physical examination was suggestive of Kaposi sarcoma); and an active herpes simplex virus type 2 genital infection. Additionally, the patient reported a history of high-risk sexual behaviours before her current 5-year relationship.

Blood work at readmission showed positive HIV serology with a CD4 count of 0.01 (normal range $0.43-1.69$ ) $\times 10^{9} / \mathrm{L}$ and a viral load of 679110 copies/mL. Given the patient's hypersensitivity reactions to sulfonamides and clindamycin, she was discharged with atovaquone for $P$. jirovecii pneumonia. Two weeks later, the patient presented to her scheduled appointment with the infectious disease service, at which time her symptoms were improving and antiretroviral therapy was begun.

\section{Discussion}

The rapid spread of information about COVID-19 has yet to be fully assimilated into the established illness scripts clinicians use in everyday patient care. ${ }^{1,2}$ In spite of our best efforts, it was diagnostic error that led to our delay in diagnosis.

\section{Diagnostic error}

Diagnostic error is the failure to establish an accurate or timely diagnosis. ${ }^{3}$ It has been viewed as an extension of cognitive failure, in conjunction with "no-fault" and "system-related" errors. ${ }^{3}$ Cognitive errors relate to a physician's knowledge, clinical acumen and problem-solving skills. This article focuses primarily on the cognitive components of diagnostic error with special emphasis assigned to premature diagnostic closure, which is an important cause of diagnostic error in the field of internal medicine. ${ }^{3}$ Defined as the failure to consider other possibilities once the initial diagnosis is proposed, premature diagnostic closure laid the foundation for our delay in diagnosis.

\section{Cognitive errors}

\section{Symptomatology}

The clinical suspicion for COVID-19 in our patient arose from her symptom profile, laboratory investigations and imaging findings, despite the absence of confirmatory testing. Clinically, our patient demonstrated the 4 most prevalent manifestations of COVID-19: fever ( $88.7 \%$ of patients), cough (57.6\%), dyspnea $(45.6 \%)$ and fatigue (29.4\%)., ${ }^{4,5}$ Despite the lack of specificity of these symptoms, this symptomology was the provider team's first step toward misdiagnosis. A more comprehensive history at this stage would have revealed the patient's long-standing constitutional symptoms, and would likely have resulted in a different diagnostic workup.

\section{Investigations}

Further diagnostic closure ensued once the results of the patient's initial investigations were returned. The 4 most common biomarkers associated with COVID-19 are hypoalbuminemia ( $75.8 \%$ of patients), elevated CRP (58.3\%), elevated LDH (57.0\%) and lymphopenia (43.1\%), ${ }^{5}$ all of which our patient demonstrated. However, these markers, like her symptoms, are nonspecific. Furthermore, our patient's chest radiograph showed bilateral interstitial infiltrates, which are seen in $72.9 \%$ of patients with a diagnosis of COVID-19. ${ }^{5}$ Importantly, however, the chest radiograph findings in COVID-19 are also nonspecific and share common radiologic features with nearly all viral pneumonias. ${ }^{6}$ In contrast to these investigations, the PCR assay employed by our facility shows excellent sensitivity (95\%) and specificity (> 99\%) when adequate viral load and proper sampling technique are achieved, yet 3 negative PCRs failed to redirect our diagnostic pathway.

\section{Computed tomography}

In order to rule out other causes and obtain diagnostic clarity, we performed CT of the chest because of its high negative predictive value $(99.0 \%-99.9 \%)^{7}$ in countries with a low prevalence of disease, such as Canada. The most common CT findings in COVID-19 are diffuse bilateral ground-glass opacities (83.3\%); ${ }^{8}$ however, this pattern is associated with numerous diseases including other viral pneumonias, ${ }^{6}$ heart failure and fungal infections (classically, $P$. jirovecii pneumonia). As such, it should be noted that in low-prevalence countries, the positive predictive value of this finding for COVID-19 ranges from $1.5 \%$ to $8.3 \% ;^{7}$ for this reason, a positive chest CT test result in isolation does not warrant diagnostic closure.

\section{No-fault and system-related errors}

Although our delayed diagnosis was driven primarily by cognitive errors, it should be noted that no-fault errors and systemrelated factors were also at play. No-fault errors describe mistakes that were unforeseeable and unavoidable, whereas system-related errors describe the failure of policies and infrastructure intended to facilitate routine health care. ${ }^{3}$ The primary no-fault error was the lack of information available on COVID-19, especially pertaining to disease prevalence, diagnosis and transmission. This no-fault error was attributable to the novelty of the disease and was particularly evident early in the pandemic, when Canada was approaching its peak number of daily cases. The system-related error most pertinent to our case was the lack of standardization in the training and orientation provided to front-line workers during the pandemic. This system-related 
error refers to clinicians not being made aware of correct processes, policies, or procedures ${ }^{3}$ and it stemmed from the lack of standardized practices early in the pandemic, with a constantly evolving understanding of COVID-19 and conflicting inputs from both the medical and public media. The lack of unified direction from a single, reliable source or regulatory body translated to the inaccurate interpretation of symptoms and investigations in the setting of suspected COVID-19. Furthermore, our initial diagnostic assessment was likely limited by the rapidly changing protocols and guidelines regarding personal protective equipment and exposure time, which led us to spend less time on the history and physical examination than we normally would have.

\section{Reflection}

Given the patient's characteristic symptomatology, blood work and radiographic features, COVID-19 remained the leading differential diagnosis until bronchoscopy results were returned (this test is rarely performed for COVID-19), despite 3 negative PCR results. The series of events that impeded time to diagnosis exemplifies multiple cognitive errors: overestimating the importance of the results of some investigations, faulty history-taking and physical examination and, most importantly, premature diagnostic closure. In hindsight, our patient illustrates a classic presentation of $P$. jirovecii pneumonia in an HIV-infected person, evidenced by the clinical manifestations (fever, cough, dyspnea and bilateral crackles), laboratory findings (lymphopenia), characteristic radiographic findings (symmetric ground-glass opacities) and concurrent AIDS-defining illnesses. ${ }^{9}$ After the right diagnosis was made, we reflected on the case and concluded that the delay in diagnosis was fuelled by premature diagnostic closure related to COVID-19, which led to inadequate history-taking and physical examination at the time of initial presentation. This reflection was a valuable exercise in continuing education, as it offered us insight into the diagnostic thought process, with opportunities for meaningful self-feedback.

\section{Conclusion}

Clinicians will be expected to adapt to the pandemic by incorporating COVID-19 into their existing illness scripts for common presentations, including fever, cough and dyspnea. We must critically review patients throughout the diagnostic workup of COVID-19, particularly in the absence of a confirmatory result.

\section{References}

1. Yousefzai R, Bhimaraj A. Misdiagnosis in the COVID era: when zebras are everywhere, don't forget the horses. JACC Case Rep 2020;2:1614-9.

2. Fang H, Xingfei P, Yingwei Q, et al. Be aware of misdiagnosis: Influenza A H1N1 in a pregnant patient with suspected COVID-19. Int J Gynaecol Obstet 2020;150:119-21.

3. Graber ML, Franklin N, Gordon R. Diagnostic error in internal medicine. Arch Intern Med 2005;165:1493-9.

4. Guan W-J, Ni Z-Y, Hu Y, et al. Clinical characteristics of coronavirus disease 2019 in China. N Engl J Med 2020;382:1708-20.

5. Rodriguez-Morales AJ, Cardona-Ospina JA, Gutiérrez-Ocampo E, et al.; Latin American Network of Coronavirus Disease 2019-COVID-19 Research (LANCOVID-19). Clinical, laboratory and imaging features of COVID-19: a systematic review and meta-analysis. Travel Med Infect Dis 2020;34:101623.

6. Koo HJ, Lim S, Choe J, et al. Radiographic and CT features of viral pneumonia. Radiographics 2018;38:719-39.

7. Kim H, Hong $\mathrm{H}$, Yoon $\mathrm{SH}$. Diagnostic performance of $\mathrm{CT}$ and reverse transcriptase polymerase chain reaction for coronavirus disease 2019: a meta-analysis. Radiology 2020;296:E145-55.

8. Bao C, Liu X, Zhang H, et al. Coronavirus disease 2019 (COVID-19) CT findings: a systematic review and meta-analysis. J Am Coll Radiol 2020;17:701-9.

9. Huang L, Cattamanchi A, Davis JL, et al.; International HIV-associated Oppor tunistic Pneumonias (IHOP) Study; Lung HIV Study. HIV-associated Pneumocystis pneumonia. Proc Am Thorac Soc 2011;8:294-300.

\section{Competing interests: None declared.}

This article has been peer reviewed.

The authors have obtained patient consent.

Affiliation: Division of General Internal Medicine, McMaster University, Hamilton, Ont.

Contributors: All of the authors contributed to the conception of the work. Brandon Budhram and Alexandra Kobza contributed to designing the work and drafting the manuscript. Brandon Budhram acquired the data. All of the authors revised the manuscript critically for important intellectual content, gave final approval of the version to be published and agreed to be accountable for all aspects of the work.

Correspondence to: Brandon Budhram, brandon.budhram@medportal.ca

The section Cases presents brief case reports that convey clear, practical lessons. Preference is given to common presentations of important rare conditions, and important unusual presentations of common problems. Articles start with a case presentation (500 words maximum), and a discussion of the underlying condition follows ( 1000 words maximum). Visual elements (e.g., tables of the differential diagnosis, clinical features or diagnostic approach) are encouraged. Consent from patients for publication of their story is a necessity. See information for authors at www.cmaj.ca. 